\title{
Secondary Metabolites from Edible and Medicinal Mushrooms as Molecular Therapy for Prostate Cancer
}

\author{
Ben-Zion Zaidman, ${ }^{1,2}$ Solomon P. Wasser, ${ }^{2}$ E Jamal A. Mahajna $^{1}$ \\ ${ }^{1}$ Migal-Galilee Technology Center, Cancer Drug Discovery Program, P.O. Box 831, Kiryat Shmona, \\ 11016, Israel; ${ }^{2}$ Institute of Evolution, University of Haifa, Mount Carmel, Haifa 31905, Israel
}

Prostate cancer $(\mathrm{PCa})$ is the second leading cause of death for Western men. Primary $\mathrm{PCa}$ is hormone dependent - that is, it is manageable by hormonal therapy. However, it rapidly develops to hormonerefractory tumors because of the accumulation of mutations in the androgen receptor (AR) or to the acquisition of alternative cellular pathways that support proliferation and inhibit apoptosis of $\mathrm{PCa}$ in androgen-independent mechanisms.

Whereas $\mathrm{PCa}$ is very common in western countries, its levels are very low in some Asian countries. Several reports linked Eastern diets and cancer occurrence, especially for $\mathrm{PCa}$. The implication of several mushrooms in the $\mathrm{PCa}$ prevention in Asia is of special interest. Over the last two to three decades, scientific and medical studies have been carried out in Japan, China, Korea and, more recently, the US that have increasingly demonstrated the potent and unique health enhancing properties of compounds extracted from a range of medicinal mushrooms.

It is well established that medicinal mushrooms possess a variety of health-promoting qualities and represent a potential source of pharmaceuticals for a number of diseases, including cancer. Although most of the attention to their anticancer activity revolved around the activity of high-molecular-weight polysaccharides, their mechanism of action is yet to be determined.

Our focus is in low-molecular-weight secondary metabolites with a well defined mechanism of action targeting the expression and function of the $\mathrm{AR}$ as a potential therapy for $\mathrm{PCa}$. These modulators might interfere with ligand binding, DNA binding, AR cross-talk with co-activators, and the cell transcription machinery. Reducing AR expression or function to a critical level would not only slow the growth of $\mathrm{PCa}$ cells, but also result in apoptosis. We hypothesize that many mushroom secondary metabolites are excellent candidates for AR modulators. This suggestion is supported by four decades of research on anticancer activities of mushroom extracts and isolated compounds. A possibility exists that the pharmacophoric structural features of some of these compounds are consistent with quantitative structure-activity relationship (QSAR) models and AR binding studies.

Our proliferation and transcriptional assay initial results also indicated the presence of such active metabolites. A total of 220 mushroom extracts were screened. We used the stable cell line, MDA-kb2, carrying the luciferase gene fused to MMTV promoter, for screening of androgen agonists and antagonists. Using this system, one can measure anti-androgenic activity by measuring the decrease in reporter activity, caused by the appropriate compounds on DHTinduced luciferase activity. Eight extracts showed anti-androgenic activity, reducing DHT-induced luciferase activity by up to $64.8 \%$, including extracts from Coprinus comatus (O.F.Müll.) S.F.Gray. Of special interest are those extracts that can also inhibit androgen-dependent $\mathrm{LNCaP}$ cell proliferation. Cell viability assays demonstrated that ethanol extracts from C. comatus selectively inhibit LNCaP cells.

Data revealing the effect of selected active mushroom extracts on the activity and expression of AR and PSA will be presented. Furthermore, the effects of selected mushroom extracts on apoptosis and cell cycle progression will be demonstrated. In addition, we will use chemical separation to obtain active fractions aiming isolation, identification, and structural elucidation of active moieties. 